\title{
Circulating cytokines in predicting development of severe acute pancreatitis
}

\author{
Anne Nieminen ${ }^{1 *}$, Mikael Maksimow³, ${ }^{2,3}$ Panu Mentula1, Lea Kyhälä', Leena Kylänpää', Pauli Puolakkainen', \\ Esko Kemppainen ${ }^{1}$, Heikki Repo ${ }^{4}$ and Marko Salmi ${ }^{2,3,5}$
}

\begin{abstract}
Introduction: Severe acute pancreatitis (AP) is associated with high morbidity and mortality. Early prediction of severe AP is needed to improve patient outcomes. The aim of the present study was to find novel cytokines or combinations of cytokines that can be used for the early identification of patients with AP at risk for severe disease.

Methods: We performed a prospective study of 163 nonconsecutive patients with AP, of whom 25 had severe AP according to the revised Atlanta criteria. Admission serum levels of 48 cytokines and growth factors were determined using Bio-Plex Pro Human Cytokine Assay 21-plex and 27-plex magnetic bead suspension panels. Admission plasma levels of C-reactive protein (CRP), creatinine and calcium were measured for comparison. In subgroup analyses, we assessed the cytokine profiles of patients with severe AP $(n=14)$ who did not have organ dysfunction (OD) upon admission (modified Marshall score $<2$ ).
\end{abstract}

Results: Of 14 cytokines elevated in the severe AP group, interleukin 6 (IL-6) and hepatocyte growth factor (HGF) levels were independent prognostic markers of severe AP. IL-6, HGF and a combination of them predicted severe AP with sensitivities of $56.0 \%, 60.0 \%$ and $72.0 \%$, respectively, and specificities of $90.6 \%, 92.8 \%$ and $89.9 \%$, respectively. The corresponding positive likelihood ratio (LR+) values were 5.9, 8.3 and 7.1, respectively. The predictive values of CRP, creatinine and calcium were comparable to those of the cytokines. In subgroup analyses of patients with severe AP and without OD upon admission, we found that IL-8, HGF and granulocyte colony-stimulating factor (G-CSF) levels predicted the development of severe AP, with G-CSF being the most accurate cytokine at a sensitivity of $35.7 \%$, a specificity of $96.1 \%$ and a LR+ of 9.1 .

Conclusions: IL-6 and HGF levels upon admission have prognostic value for severe AP which is similar to levels of CRP, creatinine and calcium. Although IL-6 and HGF, as either single or combined markers, were not perfect in identifying patients at risk for severe AP, the possibility that combining them with novel prognostic markers other than cytokines might improve prognostic accuracy needs to be studied. The accuracy of IL-8, HGF and G-CSF levels in predicting severe AP in patients without clinical signs of OD upon admission warrants larger studies.

\section{Introduction}

Acute pancreatitis (AP) is usually a mild disease, resolving within days. Severe AP, as defined according to the revised Atlanta criteria [1], accounts for about $20 \%$ of cases. The mortality rate in severe AP ranges from $9 \%$ to $24 \%[2,3]$ and can be as high as $47 \%$ to $69 \%$ among patients who develop multiple organ dysfunction syndrome $[4,5]$.

In $\mathrm{AP}$, premature activation of trypsin within pancreatic acinar cells causes pancreatic autodigestion, leading to a

\footnotetext{
* Correspondence: anne.k.nieminen@hus.fi

'Department of Surgery, Helsinki University Central Hospital, POB 340, Helsinki 00029 HUS, Finland

Full list of author information is available at the end of the article
}

local inflammatory process. This process is characterized by the release of pro- and anti-inflammatory cytokines and other inflammatory mediators, which recruit neutrophils, monocytes and lymphocytes into the pancreas. In severe AP, the local inflammatory process is amplified and spreads through the circulation throughout the body, resulting in a systemic inflammatory response $[6,7]$. Systemic inflammation is thought to contribute to the development of organ dysfunction (OD), which may be transient ( $<48$ hours) or persistent ( $>48$ hours). The latter is associated with a $35 \%$ to $50 \%$ mortality rate $[8,9]$. Accurate prediction of persistent OD is needed 
because these patients will benefit from general supportive care, including early fluid resuscitation, in the ICU $[10,11]$.

Although levels of a variety of cytokines, determined upon admission to the hospital, may predict the course of AP [12-15], no cytokine has proved to be useful enough to be incorporated into routine clinical use. Furthermore, to the best of our knowledge, no attempt has been made to discriminate the predictive power of cytokines in patients with OD upon admission from those who are bound to develop OD during hospitalization.

In this study, we analyzed 48 different cytokines in a total of 25 patients with severe AP to determine, upon admission to hospital, novel markers specific for such patients. We used plasma levels of $\mathrm{C}$-reactive protein (CRP), creatinine and calcium as conventional biomarkers. In further analyses of the data, we excluded patients with modified Marshall scores $\geq 2$, whom, we reasoned, already had OD upon admission, compared with the respective patients with modified Marshall scores $<2$, who developed OD later during hospitalization.

\section{Methods}

\section{Patients and definitions}

In this prospective study conducted at the Helsinki University Central Hospital between June 2003 and January 2008, we assessed 163 nonconsecutive patients with AP admitted within 72 hours of the onset of symptoms. Patients with the signs of chronic pancreatitis were excluded.

AP was diagnosed if two of the following three features were observed: typical abdominal upper epigastric pain, serum or plasma amylase level at least three times greater than the upper limit of normal and characteristic findings of AP on transabdominal ultrasonography, computed tomography or magnetic resonance imaging. The severity of AP was categorized retrospectively according to the revised Atlanta classification system as mild, moderately severe (local complication or transient OD) or severe (persistent OD) [1]. Two patients with severe AP were referred to Helsinki University Central Hospital because they needed to be admitted to the ICU.

The Marshall score [16] was calculated according to the method described by Banks et al. [1] to assess the presence of OD upon admission. Thus, OD was deemed to be present if the modified Marshall score was $\geq 2$ for one of the three organ systems (respiratory, renal or cardiovascular). A flowchart of the patient distribution is presented in Figure 1.

All patients, or their next of kin, gave their informed consent to participate. The Ethics Committee of the Helsinki University Central Hospital Department of Surgery approved the study.

\section{Samples and cytokine measurements}

Serum samples were collected upon admission to the hospital and stored at $-70^{\circ} \mathrm{C}$ until used for analyses. Each sample $(20 \mu \mathrm{l})$ was studied by magnetic bead suspension array using the Bio-Plex Pro Human Cytokine 21- and 27-plex panels (Bio-Rad Laboratories, Hercules, CA, USA) according to the manufacturer's instructions except that the assay reagents were used at half of their recommended concentrations. The 21-plex panel contains interleukin $1 \alpha$ (IL-1 $\alpha)$, IL-2 receptor $\alpha$ (IL-2R $\alpha)$, IL-3, IL-12p40, IL-16, IL-18, cutaneous T-cell attracting chemokine, growth-regulated oncogene $\alpha$, hepatocyte growth factor (HGF), interferon $\alpha 2$ (IFN- $\alpha 2)$, leukemia inhibitory factor (LIF), monocyte chemotactic protein 3 (MCP-3), macrophage colony-stimulating factor (M-CSF), macrophage migration inhibitory factor, monokine induced by IFN- $\gamma, \beta$-nerve growth factor, stem cell factor, stem cell growth factor $\beta$, stromal cell-derived factor $1 \alpha$, tumor necrosis factor $\beta$ (TNF- $\beta$ ) and TNF-related apoptosis inducing ligand. The 27-plex contains IL-1 $\beta$, IL-1 receptor antagonist, IL-2, IL-4, IL-5, IL-6, IL-7, IL-8, IL-9, IL-10, IL-12p70, IL13, IL-15, IL-17A, basic fibroblast growth factor, eotaxin, granulocyte colony-stimulating factor (G-CSF), granulocytemacrophage colony-stimulating factor (GM-CSF), IFN- $\gamma$, IFN- $\gamma$-induced protein 10 , monocyte chemotactic protein 1 (MCP-1), macrophage inflammatory protein $1 \alpha$ (MIP-1 $\alpha$ ), MIP-1 $\beta$, platelet-derived growth factor $B B$, regulated on activation, normal $\mathrm{T}$ cell expressed and secreted (that is, RANTES), TNF- $\alpha$ and vascular endothelial growth factor. The samples were analyzed using the Bio-Plex 200 System, and the results were calculated using Bio-Plex Manager 6.0 software (Bio-Rad Laboratories).

No measurable values for IFN- $\alpha 2$ were obtained from any sample, and thus this cytokine was excluded from data analyses. Also, the majority of the values for LIF, eotaxin, GM-CSF, IL-1 $\alpha$, IL-3, IL-12p40, IL-15 and TNF- $\beta$ were below the detectable limit, and therefore these cytokines were scored on a dichotomous scale of detectable or undetectable value. The persons doing the cytokine measurements were unaware of the clinical status of the patients.

Plasma levels of CRP (normal reference range $<10 \mathrm{mg} / \mathrm{L}$ ), creatinine (50 to $90 \mu \mathrm{mol} / \mathrm{L}$ ) and calcium (2.15 to 2.51 $\mathrm{mmol} / \mathrm{L}$ ) were determined in accordance with our hospital's laboratory routine.

\section{Statistical analysis}

Statistical analysis was performed using IBM SPSS version 19 statistical software (SPSS, Chicago, IL, USA). The results are given as medians with ranges and interquartile ranges (IQR). Spearman's rank correlation was used to test correlations between two continuous variables. For univariate analysis, comparisons between groups were made using the Mann-Whitney $U$ test. The level of significance was adjusted using the Bonferroni method by dividing the 


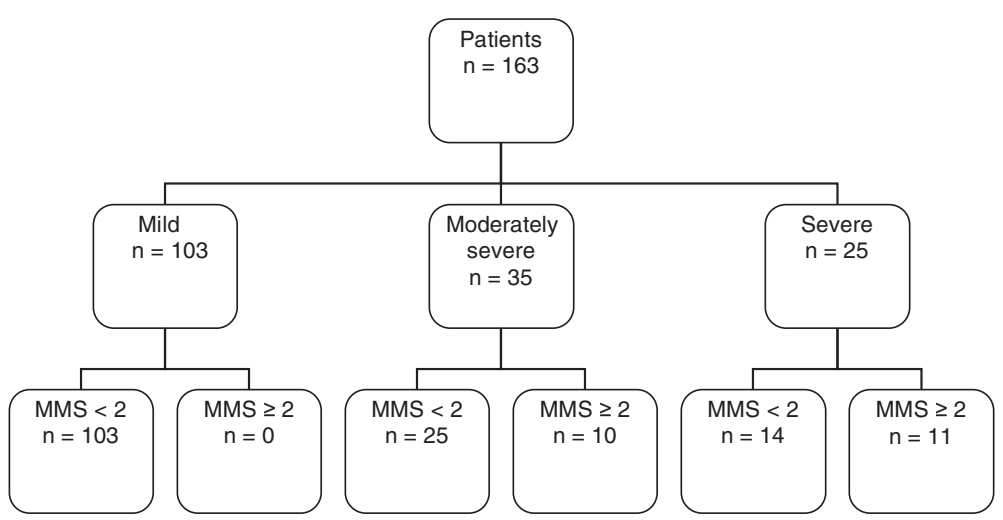

Figure 1 Patient classifications according to modified Marshall score [20] and revised Atlanta criteria [1] upon admission. MMS,

Modified Marshall score.

significance level 0.05 by the number of simultaneous tests. After univariate analysis, parameters were entered into multivariate analysis, and logistic regression analysis was performed to identify independent markers predictive of persistent OD. In the post hoc analysis, we determined clinically optimal cutoff values for each cytokine using receiver operating characteristic (ROC) curves, with corresponding sensitivities, specificities, positive likelihood ratios $(\mathrm{LR}+)$, negative likelihood ratios (LR-) and diagnostic odd ratios (DORs) with 95\% confidence intervals [17].
The DOR is the ratio of the odds of positive test results (OD) among patients with OD to the odds of a positive test result (OD) among the patients without OD. The higher the value, the better the discriminatory test performance is [18]. The clinically optimal cutoff value was defined as the point on the curve where the number or false positives is as low as possible (specificity $\geq 90 \%$ ), with the maximum sensitivity, to avoid overtreatment of patients in the ICU. Areas under the ROC curve were also calculated.

Table 1 Characteristics of the patients ${ }^{a}$

\begin{tabular}{|c|c|c|c|c|c|c|c|}
\hline \multirow[t]{2}{*}{ Characteristics } & \multirow{2}{*}{$\begin{array}{l}\text { All patients } \\
(N=163)\end{array}$} & \multirow{2}{*}{$\begin{array}{l}\text { Mild or moderately } \\
\text { severe AP }(n=138)\end{array}$} & \multirow[t]{2}{*}{$P$-value ${ }^{b}$} & \multicolumn{4}{|c|}{ Severe AP $(n=25)$} \\
\hline & & & & All $(N=25)$ & MMS $<2(n=14)$ & $P$-value & MMS $\geq 2(n=11)$ \\
\hline Men & $120(73.6)$ & $96(69.6)$ & 0.006 & $24(96.0)$ & $13(92.9)$ & NS & $11(100.0)$ \\
\hline Age, years & 48 (18 to 87$)$ & 49 (18 to 87$)$ & NS & 43 (29 to 81$)$ & 41 (29 to 81$)$ & NS & 51 (29 to 69) \\
\hline \multicolumn{8}{|l|}{ Etiology } \\
\hline Alcohol & $113(69.3)$ & $91(65.9)$ & 0.028 & $22(88.0)$ & 11 (78.6) & NS & $11(100)$ \\
\hline Biliary & $35(21.5)$ & $34(24.6)$ & 0.021 & $1(4.0)$ & $1(7.1)$ & NS & 0 \\
\hline Other cause & $11(6.7)$ & $9(6.5)$ & NS & $2(8.0)$ & $2(14.3)$ & NS & 0 \\
\hline Idiopathic & $4(2.5)$ & $4(2.9)$ & NS & 0 & 0 & NS & 0 \\
\hline Onset of symptoms, hr & 24 (1 to 72$)$ & 24 (1 to 72$)$ & NS & 24 (3 to 72 ) & 18 (3 to 48$)$ & $<0.001$ & 48 (24 to 72$)$ \\
\hline C-reactive protein, $\mathrm{mg} / \mathrm{L}$ & 19 (3 to 435$)$ & 16 (3 to 426) & 0.002 & 94 (3 to 435) & 30 (3 to 229) & $<0.001$ & 294 (22 to 435$)$ \\
\hline Creatinine, $\mu \mathrm{mol} / \mathrm{L}$ & $64(31$ to 1086$)$ & 62 (31 to 313 ) & $<0.001$ & 92 (47 to 1086) & 68 (47 to 147) & $<0.001$ & 248 (92 to 1086) \\
\hline Calcium, mmol/L & $2.2(1.27$ to 2.73$)$ & 2.23 (1.27 to 2.73$)$ & $<0.001$ & 1.86 (1.39 to 2.30$)$ & 2.04 (1.49 to 2.30$)$ & 0.013 & 1.67 (1.39 to 2.17$)$ \\
\hline Length of hospital stay, days & $6(1$ to 93$)$ & $5(1$ to 41$)$ & $<0.001$ & 26 (1 to 93$)$ & 28 (1 to 93$)$ & NS & 17 (1 to 49$)$ \\
\hline Mechanical ventilation & $25(15.3)$ & $2(1.4)$ & $<0.001$ & $23(92.0)$ & $13(92.9)$ & NS & $10(90.9)$ \\
\hline Renal replacement therapy & $16(9.8)$ & 0 & $<0.001$ & $16(64.0)$ & $7(50.0)$ & NS & $9(81.8)$ \\
\hline OD within 24 hours & $30(18.4)$ & $10(7.2)$ & $<0.001$ & $20(80.0)$ & $9(64.3)$ & NS & $11(100)$ \\
\hline OD on days 1 to 7 & $5(3.1)$ & 0 & $<0.001$ & $5(20.0)$ & $5(37.5)$ & NS & $0(0)$ \\
\hline Mortality & $8(4.9)$ & $1(0.7)$ & 0.014 & $7(28.0)$ & $2(14.3)$ & NS & $5(45.5)$ \\
\hline
\end{tabular}

${ }^{\mathrm{a}} \mathrm{AP}$, Acute pancreatitis; MMS, Modified Marshall score; NS, Not significant; OD, organ dysfunction. ${ }^{\mathrm{b}}$ Significance of the difference between mild or moderately severe AP $(n=138)$ and severe AP $(n=25)$. The severe AP group is categorized into two subgroups: admission modified Marshall Score $<2$ or $\geq 2$. Data are median (range) or $n(\%)$. 


\section{Results}

\section{Patients}

The characteristics of the patients are shown in Table 1. The severity of AP was classified as mild in 103 patients (63\%), moderately severe in 35 patients (17\%) and severe in 25 patients (15\%). All of the patients with severe AP developed persistent OD and required invasive mechanical ventilation and/or renal replacement therapy.

OD was present upon admission in 21 patients (13\%). Ten of these cases were transient OD, which responded rapidly to treatment and resolved within 48 hours. In these cases, the severity of AP was classified as moderately severe. Eleven patients with severe AP had OD upon admission, and another nine patients developed OD within 24 hours after admission. Five patients developed OD within 1 to 7 days after admission. There were no cases of new-onset OD after the first week of treatment.

The overall mortality rate was $4.9 \%$. Seven (28\%) of the twenty-five patients with severe AP died. Two of them were not admitted to the ICU because of their old age and comorbidities, and they died within 48 hours. One patient with moderately severe AP died as a result of carbon dioxide retention.

\section{Cytokine profiles of patients with severe acute pancreatitis}

The levels of 14 of 47 cytokines measured were significantly higher in the severe AP group than in the mild and moderately severe AP groups $(P<0.001$ after Bonferroni adjustment) (Table 2). The corresponding differences in the levels of the other 33 cytokines were not significant (Table 3).

By stepwise forward logistic regression analysis of the 14 cytokines, we identified IL-6 $(P=0.006)$ and HGF $(P<0.001)$ as independent prognostic markers of severe AP. Many of the other 14 cytokines (M-CSF, HGF, IL-8, MCP-1 and G-CSF) had a strong positive Spearman's correlation with IL-6 $(r>0.6)$ (Table 4). M-CSF, IL-8, MCP-1 and G-CSF did not improve the explanatory power of the regression model and thus were excluded from the stepwise analysis.

IL-6 and HGF levels predicted severe AP with sensitivities of $48.0 \%$ and $60.0 \%$, respectively, and specificities of $93.5 \%$ and $92.8 \%$, respectively (Table 5 ). In the combined regression model of IL- 6 and HGF, sensitivity was improved to $72.0 \%$ and specificity remained high at $89.9 \%$. The LR+ values for IL-6, HGF and the combined model were 7.4, 8.3 and 7.1, respectively, and the DOR values were 13.2, 19.8 and 22.8, respectively. None of the differences between IL-6, HGF and the combined model were statistically significant (Table 5 ). Additional file 1 shows the diagnostic performance of all of the 14 cytokines that could differentiate mild or moderately severe AP from severe AP.

The discriminatory power of plasma calcium $<1.91$ $\mathrm{mmol} / \mathrm{L}$, creatinine $>141 \mu \mathrm{mol} / \mathrm{L}$ and CRP $>227 \mathrm{mg} / \mathrm{L}$ was comparable to that of IL-6 and HGF (Table 5).

Table 2 The 14 cytokines that differed between mild or moderately severe acute pancreatitis patients and severe acute pancreatitis patients ${ }^{\mathrm{a}}$

\begin{tabular}{|c|c|c|c|c|c|c|}
\hline \multirow[b]{2}{*}{ Cytokines } & \multicolumn{3}{|c|}{ All patients $(N=163)$} & \multicolumn{3}{|c|}{ Admission MMS $<2(n=142)$} \\
\hline & $\begin{array}{l}\text { Mild or moderately } \\
\text { severe AP }(n=138)\end{array}$ & $P$-value ${ }^{\mathrm{b}}$ & Severe $(n=25)$ & $\begin{array}{l}\text { Mild or moderately } \\
\text { severe AP }(n=128)\end{array}$ & $P$-value ${ }^{b}$ & Severe AP $(n=14)$ \\
\hline G-CSF & $119.7(66.6-198.2)$ & $<0.001$ & $260.2(132.5$ to 1011.8$)$ & 118.41 (66.6 to 174.3 ) & 0.0007 & $234.0(151.2$ to 531.1$)$ \\
\hline GRO-a & $58.8(35.7-92.3)$ & $<0.001$ & $128.2(98.4$ to 169.1$)$ & 54.5 (33.2 to 82.5$)$ & 0.002 (NS) & 118.5 (62.4 to 158.4) \\
\hline HGF & $1055.5(764.7-1730.6)$ & $<0.001$ & 3613.0 (2055.3 to 6348.8) & 989.6 (753.6 to 1501.6$)$ & $<0.001$ & 2202.6 (1554.3 to 3305.5) \\
\hline IL-2Ra & $214.2(150.6-316.7)$ & $<0.001$ & 483.3 (385.6 to 673.3 ) & 209.5 (145.5 to 290.4$)$ & 0.0015 (NS) & 449.7 (228.1 to 504.3$)$ \\
\hline IL-6 & $59.7(15.3-202.1)$ & $<0.001$ & 428.9 (138.5 to 1796.3$)$ & 49.9 (14.7 to 185.6$)$ & 0.0013 (NS) & 234.5 (66.2 to 1931.3$)$ \\
\hline IL-8 & $26.6(19.3-41.9)$ & $<0.001$ & 82.4 (46.4 to 115.6$)$ & 25.41 (18.0 to 37.6$)$ & $<0.001$ & 59.7 (32.0 to 102.8) \\
\hline IL-18 & $139.6(91.6-187.1)$ & $<0.001$ & 202.8 (151.8 to 305.4$)$ & 131.5 (90.7 to 181.5$)$ & 0.062 (NS) & 162.4 (135.3 to 201.6) \\
\hline $\mathrm{LIF}^{\mathrm{C}}$ & $0(0)$ & $<0.001$ & 1 (0 to 1$)$ & $0(0)$ & 0.002 (NS) & $1(0$ to 1$)$ \\
\hline M-CSF & $14.5(8.1-28.1)$ & $<0.001$ & $40.2(18.6$ to 68.6$)$ & $12.4(5.5$ to 21.5$)$ & 0.007 (NS) & 28.7 (12.2 to 52.0) \\
\hline MCP-1 & $49.0(21.0-111.2)$ & $<0.001$ & 125.0 (64.6 to 199.6$)$ & 47.6 (30.0 to 100.6) & 0.032 (NS) & 92.1 (36.3 to 185.2) \\
\hline MCP-3 & $17.4(0.57-53.6)$ & $<0.001$ & 54.58 (24.3 to 98.7$)$ & $16.3(0.2$ to 47.9$)$ & 0.006 (NS) & $52.0(16.5$ to 102.1$)$ \\
\hline$\beta-N G F$ & $5.79(3.65-9.1)$ & $<0.001$ & 11.3 (7.4 to 15.9$)$ & 5.5 (3.4 to 8.5 ) & 0.007 (NS) & 10.4 (4.5 to 15.5$)$ \\
\hline SCF & $114.1(85.2-147.0)$ & 0.0008 & $155.8(109.2$ to 256.0$)$ & 111.6 (82.0 to 143.3 ) & 0.012 (NS) & 148.5 (107.3 to 218.9$)$ \\
\hline SDF-1a & $87.6(53.5-151.5)$ & $<0.001$ & 163.6 (104.4 to 226.3$)$ & 86.8 (52.8 to 148.5$)$ & 0.010 (NS) & 171.3 (85.1 to 234.8) \\
\hline
\end{tabular}

${ }^{a} \beta-N G F, \beta$-nerve growth factor; G-CSF, granulocyte colony-stimulating factor; GRO-a, Growth-related oncogene a; HGF, Hepatocyte growth factor; IL, Interleukin; IL-1Ra, Interleukin 1 receptor antagonist; IL-2Ra, Interleukin 2 receptor a; SDF, stromal derived factor; LIF, Leukemia inhibitory factor; MCP, Monocyte chemotactic protein; M-CSF, Macrophage colony-stimulating factor; SDF, Stromal derived factor; SCF, Stem cell factor; SDF, Stromal cell-derived factor IQR, interquartile range; NS, not significant. ${ }^{b}$ Level of significance is defined as $P<0.001$ (Bonferroni-adjusted). ${ }^{c} 0=$ Undetectable value, $1=$ Detectable value. Cytokine levels (pg/ml) are expressed as median (IQR). 
Table 3 Levels of 33 cytokines showing no significant difference between mild or moderately severe and severe acute pancreatitis $^{\text {a }}$

\begin{tabular}{|c|c|c|c|}
\hline Cytokines & Mild or moderately severe AP $(n=138)$ & $P$-value ${ }^{b}$ & Severe AP $(n=25)$ \\
\hline CTACK & 993.1 (746.4 to $1,556.1)$ & 0.033 (NS) & $693.3(652.5$ to $1,150.6)$ \\
\hline Eotaxin $^{c}$ & $1(0$ to 1$)$ & 0.133 (NS) & $1(0$ to 1$)$ \\
\hline FGF & $25.9(8.6$ to 44.6$)$ & 0.123 (NS) & 19.4 (0.4 to 35.2$)$ \\
\hline $\mathrm{GM}-\mathrm{CSF}^{\mathrm{C}}$ & $0(0)$ & 0.663 (NS) & $0(0$ to 1$)$ \\
\hline IFN- $\gamma$ & 99.5 (50.7 to 151.0$)$ & 0.772 (NS) & 82.4 (50.9 to 149.5$)$ \\
\hline $\mid \mathrm{P}-10$ & 379.3 (244.5 to 594.0$)$ & 0.475 (NS) & 446.2 (222.6 to 825.3$)$ \\
\hline $\mid L-1 a^{c}$ & $0(0$ to 1$)$ & 0.473 (NS) & $1(0$ to 1$)$ \\
\hline $\mathbb{I L}-1 \beta$ & 1.9 (0.8 to 3.0$)$ & 0.961 (NS) & $1.9(0.5$ to 3.4$)$ \\
\hline IL-1Ra & 121.1 (64.8 to 260.0) & 0.131 (NS) & 202.6 (70.9 to 669.8) \\
\hline $\mathrm{IL}-2$ & $7.6(2.2$ to 13.4$)$ & 0.796 (NS) & 6 to 7 (1.7 to 12.9$)$ \\
\hline $\mid L-3^{c}$ & $0(0)$ & 0.008 (NS) & $0(0$ to 1$)$ \\
\hline $\mid \mathrm{L}-4$ & $5.1(2.6$ to 7.5$)$ & 0.261 (NS) & 3.8 (2.3 to 6.4$)$ \\
\hline IL-5 & 2.7 (1.6 to 4.1$)$ & 0.091 (NS) & 1.8 (1.0 to 3.4$)$ \\
\hline $\mathbb{I L}-7$ & 5.9 (3.9 to 10.5$)$ & 0.482 (NS) & $5.2(3.9$ to 9.4$)$ \\
\hline IL-9 & 19.5 (7.3 to 37.8$)$ & 0.410 (NS) & $16.1(1.7$ to 38.7$)$ \\
\hline$\| \mathrm{L}-10$ & 8.2 (3.9 to 17.7$)$ & 0.012 (NS) & 15.8 (6.2 to 42.3$)$ \\
\hline IL-12p70 & $18.7(11.2$ to 40.4$)$ & 0.442 (NS) & 15.5 (9.0 to 36.5$)$ \\
\hline IL-12p40 & $0(0)$ & 0.691 (NS) & $0(0)$ \\
\hline $\mathbb{I L}-13$ & $7.2(3.9$ to 13.5$)$ & 0.249 (NS) & $6.5(2.6$ to 12.1$)$ \\
\hline $\mid \mathrm{L}-15^{\mathrm{c}}$ & $0(0)$ & 0.018 (NS) & 0 (0 to 1$)$ \\
\hline IL-16 & 220.2 (99.9 to 786.8 ) & 0.120 (NS) & $341.3(157.2$ to 980.1$)$ \\
\hline IL-17A & 79.8 (33.2 to 155.9$)$ & 0.084 (NS) & 45.8 (9.2 to 98.4$)$ \\
\hline MIP-1a & 6.2 (3.7 to 8.0) & 0.215 (NS) & $4.6(2.4$ to 7.1$)$ \\
\hline MIP-1 $\beta$ & 67.1 (42.6 to 90.2$)$ & 0.830 (NS) & $63.6(40.1$ to 95.8$)$ \\
\hline MIF & $695.8(348.5$ to $1,553.9)$ & 0.004 (NS) & 1461.2 (713.4 to $4,529.4)$ \\
\hline MIG & $1,415.5$ (821.0 to $2,348.2)$ & 0.021 (NS) & $2,147.5(1,257.4$ to $4,268.7)$ \\
\hline PDGF-BB & 1,183.6 (360.4 to $2,475.2$ ) & 0.063 (NS) & 583.3 (207.8 to $1,427.3)$ \\
\hline RANTES & $2,905.6(1,110.3$ to $4,511.1)$ & 0.093 (NS) & $1,877.2$ (787.5 to $3,090.9)$ \\
\hline SCGF- $\beta$ & 31452.4 (21610.3 to 46978.0$)$ & 0.004 (NS) & 47129.9 (30743.1 to 56343.2) \\
\hline TRAIL & 57.4 (33.3 to 92.0$)$ & 0.042 (NS) & 84.8 (51.8 to 122.7$)$ \\
\hline TNF-a & 20.8 (10.3 to 31.9) & 0.730 (NS) & 18.2 (9.7 to 31.5) \\
\hline TNF- $\beta^{c}$ & $0(0$ to 1$)$ & 0.187 (NS) & $0(0)$ \\
\hline VEGF & 29.9 (17.7 to 71.1$)$ & 0.436 (NS) & 23.6 (17.4 to 64.2$)$ \\
\hline
\end{tabular}

${ }^{a}$ AP, Acute pancreatitis; CTACK, Cutaneous T-cell attracting chemokine; FGF, Basic fibroblast growth factor; GM-CSF, Granulocyte-macrophage colony-stimulating factor; IFN, Interferon; IL, Interleukin; IL-1Ra, Interleukin 1 receptor antagonist; IP-10, Interferon- $\gamma$-induced protein 10; VEGF, vascular endothelial growth factor; MIF, Macrophage migration inhibitory factor; MIG, Monokine induced by interferon $\gamma ;$ MIP, Macrophage inflammatory protein; VEGF, vascular endothelial growth factor; PDGF-BB, Platelet-derived growth factor, two BB chains; RANTES, Regulated on activation, normal T cell expressed and secreted; SCGF- $\beta$, Stem cell growth factor $\beta$; TNF, Tumor necrosis factor; TRAIL, Tumor necrosis factor-related apoptosis-inducing ligand; VEGF, Vascular endothelial growth factor IQR, interquartile range; NS, Not significant. ${ }^{b}$ Level of significance is defined as $P<0.001$ (Bonferroni-adjusted). ${ }^{c} 0=$ Undetectable value, $1=$ Detectable value. Data are median (IQR).

When calcium, creatinine and CRP were added into the logistic regression analysis with IL-6 and HGF, calcium was also able to predict severe AP independently $(P=0.023)$, as were IL-6 $(P=0.025)$ and HGF $(P=0.027)$, but creatinine $(P=0.86)$ and CRP $(P=0.45)$ were not. The ROC curves for the logistic regression model of IL-6 and HGF and that for CRP are presented in
Figure 2, and their clinically optimal cutoff points are listed in Table 5.

\section{Predicting severe acute pancreatitis in patients with} modified Marshall score $<2$ upon admission

In the patients who developed persistent OD after admission to the hospital $(n=14)$, compared with patients who 
Table 4 Cytokines showing strong correlation with interleukin $6^{\mathrm{a}}$

\begin{tabular}{lll}
\hline Cytokines & Spearman's $r$ & $P$-value \\
\hline M-CSF & 0.62 & $<0.001$ \\
HGF & 0.67 & $<0.001$ \\
IL-8 & 0.67 & $<0.001$ \\
MCP-1 & 0.71 & $<0.001$ \\
G-CSF & 0.72 & $<0.001$ \\
\hline
\end{tabular}

${ }^{\mathrm{a} G-C S F}$, Granulocyte colony-stimulating factor; HGF, Hepatocyte growth factor; IL, Interleukin; MCP, Monocyte chemotactic protein.

did not $(n=128)$, the levels of IL- 8 , HGF and G-CSF were significantly higher $(P<0.001$, Bonferroni-adjusted $)$ (Table 2). We did not perform multivariate analysis in this subgroup, owing to the low number of patients. We therefore determined how many of these 14 OD patients could be identified using the same clinically optimal cutoff points that we used earlier for the whole patient cohort (Table 5). G-CSF correctly predicted severe AP in 5 of 14 patients (Table 6). IL- 8 detected one patient, and HGF identified two additional patients. Thus, the combination of these three cytokines detected eight of fourteen OD patients. The diagnostic performance of each cytokine (IL-8, HGF and G-CSF) and that of the combination are presented in Table 7.

In the subgroup of patients who developed persistent OD after admission, calcium had a sensitivity of $36.4 \%$, a specificity of $94.3 \%$, a LR+ value of 6.4 and a DOR value of 9.5. These values were similar to the respective values for G-CSF (Table 6). The sensitivity of CRP and creatinine each was $7.1 \%$, indicating poor performance in predicting OD. The ROC curves of G-CSF and CRP are presented in Figure 3.

Predicting severe acute pancreatitis in patients with admission modified Marshall scores $\geq \mathbf{2}$

When comparing the levels of 14 cytokines in the patients with transient OD $(n=10)$ or persistent OD $(n=11)$, we found that the levels of HGF were higher in the patients with persistent OD $(P=0.007)$; however, the difference was not significant after we applied the Bonferroni correction. IL- 6 and IL- 8 had P-values of 0.067 and 0.057 , respectively, and the rest of the cytokines had $P$-values $>0.1$.

\section{Discussion}

The results of our present study show that the levels of 14 of 47 cytokines were significantly higher in the severe AP group than in the patients with mild or moderately severe AP. Regression analysis, however, revealed that only IL-6 and HGF were independent predictive markers of severe AP. Both IL-6 and HGF are multifunctional cytokines that play many roles in inflammation, and the serum levels of these cytokines reflect the magnitude of the inflammatory response.

IL-6 is a proinflammatory cytokine released by macrophages in response to tissue injury, and it stimulates the synthesis of acute phase proteins (for example, CRP) in the liver. HGF stimulates mitogenesis, cell motility and matrix invasion, and thus it has a central role in angiogenesis, tumorigenesis and tissue regeneration [19]. Both IL-6 [12,20] and HGF [21,22] have been demonstrated to have prognostic value for AP upon admission. These studies, together with the results of the present study, indicating LR+ values 7.4 and 8.3 for IL-6 and HGF, respectively, for predicting severe $\mathrm{AP}$, thus give credence to the view etc., thus giving credence to the view that single cytokines such as IL-6 and HGF may not be perfect predictors of severe AP. Our results also show that the LR+ value for the combination of IL- 6 and HGF was 7.1, indicating that combining the cytokines did not improve their predictive accuracy for severe AP. One reason for this may be the strong Spearman's correlation between IL-6 and HGF, and, indeed, with many other cytokines (Table 4). Moreover, the sensitivities, specificities and $\mathrm{LR}+$ were determined using post hoc analysis in which the cutoff points were set in the same population. If the analyses had been done prospectively with preset cutoff values, the predictive power of biomarkers would most

Table 5 Diagnostic performances of interleukin 6 and hepatocyte growth factor and conventional markers in predicting severe acute pancreatitis in all patients $(N=163)^{a}$

\begin{tabular}{|c|c|c|c|c|c|c|}
\hline $\begin{array}{l}\text { Predictive marker with } \\
\text { optimal cutoff point }\end{array}$ & AUC & Sensitivity (\%) & Specificity (\%) & LR+ & LR- & DOR \\
\hline-6( & 0.81 (0.72 to 0.90$)$ & ) & ) & 5) & ) & $13.2(4.7$ to 37.3$)$ \\
\hline $\operatorname{HGF}(>3,020.1 \mathrm{pg} / \mathrm{ml})$ & 0.87 (0.81 to 0.94$)$ & 60.0 (40.7 to 0.77$)$ & 92.8 (87.1 to 96.0$)$ & 8.3 (4.2 to 16.3 ) & 0.43 (0.27 to 0.70$)$ & $19.2(6.9$ to 53 \\
\hline$L-6+H G F$ & 0.89 (0.82 to 0.96$)$ & 72.0 (52.4 to 85.7$)$ & 89.9 (83.7 to 93.9) & 7.1 (4.1 to 12.3$)$ & 0.31 (0.17 to 0.59$)$ & 22.8 (8.1 to 64.0$)$ \\
\hline CRP (>227 mg/L) & 0.692 (0.57 to 0.82 ) & 40.0 (23.4 to 59.3$)$ & 93.5 (88.0 to 96.5 ) & 6.1 (2.8 to 13.6$)$ & 0.64 (0.47 to 0.89$)$ & 9.6 (3.4 to 27.2$)$ \\
\hline Creatinine $(>141 \mu \mathrm{mol} / \mathrm{L})$ & 0.76 (0.65 to 0.88$)$ & 40.0 (23.4 to 59.3$)$ & 96.3 (91.7 to 98.4) & $10.9(4.1$ to 29.1$)$ & 0.62 (0.45 to 0.86$)$ & 17.5 (5.3 to 57.9$)$ \\
\hline Ealcium (<1.91 mmol/L) & $0.81(0.70$ to 0.91$)$ & 57.9 (36.3 to 76.9$)$ & 93.2 (87.6 to 96.4$)$ & 8.5 (4.1 to 17.8$)$ & 0.45 (0.27 to 0.77$)$ & $18.8(6.0$ to 58 \\
\hline
\end{tabular}

alL, interleukin; CRP, C-reactive protein; IL, interleukin; HGF, Hepatocyte growth factor; IL, Interleukin; AUC, Area under curve; DOR, Diagnostic odds ratio; LR-, Negative likelihood ratio; LR+, Positive likelihood ratio; $95 \%$ confidence intervals are given in parentheses. 


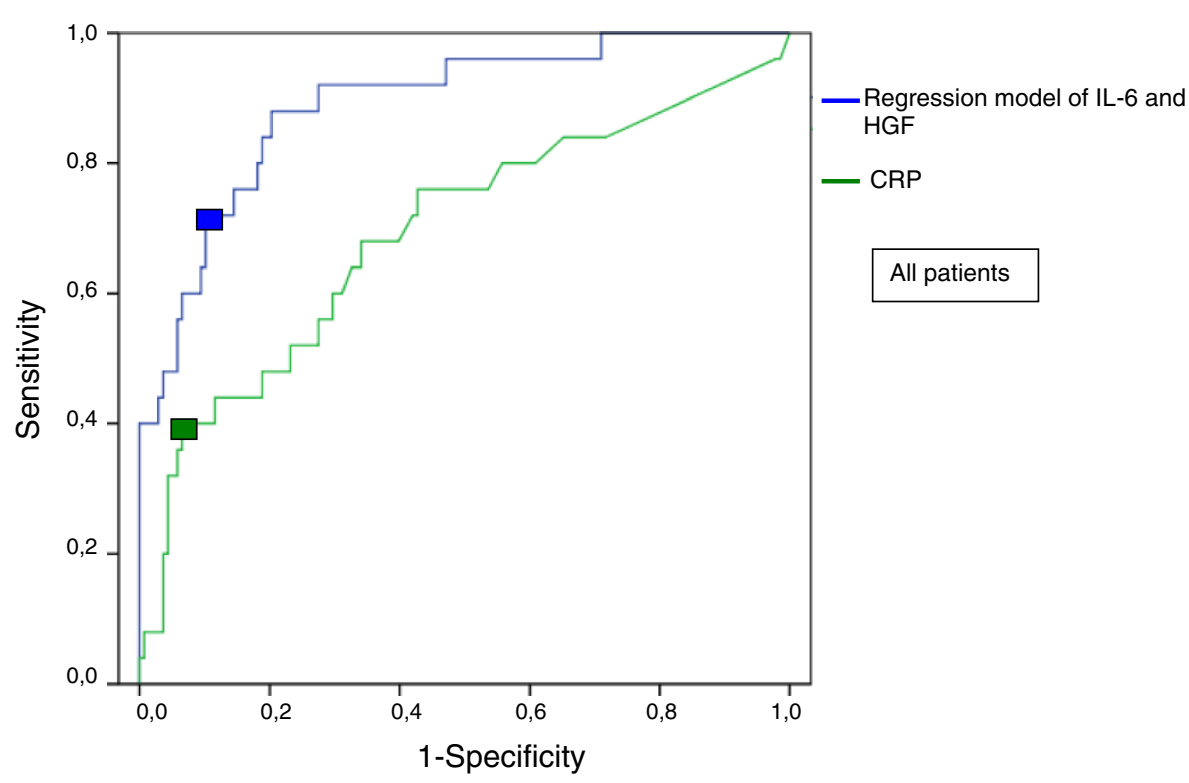

Figure 2 Receiver operating characteristic curves of C-reactive protein and interleukin 6 plus hepatocyte growth factor for prediction of severe acute pancreatitis in the entire acute pancreatitis patient cohort $(N=163)$. Boxes indicate clinically optimal cutoff points used to calculate the sensitivity and specificity of each biomarker listed in Table 5. CRP, C-reactive protein; HGF, Hepatocyte growth factor; IL, Interleukin.

probably have been even lower. Taken together, the results suggest that none of the cytokines alone or combinations of them would be useful in clinical practice, which would require $L R+$ values $>10$.

In agreement with previous studies [23-25], our results show that many severe AP patients already have OD upon presentation. Consequently, to find true prognostic markers, such patients should be excluded from further analysis. We did so in the subgroup analysis with a limited number of severe AP patients $(n=14)$. When we compared the severe AP groups with admission modified Marshall scores $<2$ or $\geq 2$, we found that the latter group had had symptoms significantly longer and had significantly higher CRP and creatinine levels upon admission, but otherwise no other clinical marker or symptom differed between the groups. In the group of severe AP patients

Table 6 Performance of biomarkers to predict severe acute pancreatitis in patients with admission modified Marshall Score $<2(N=14)^{\mathrm{a}}$

\begin{tabular}{|c|c|c|c|c|c|c|}
\hline $\begin{array}{l}\text { Severe AP } \\
\text { patients }\end{array}$ & IL-8 $>88.1 \mathrm{pg} / \mathrm{ml}$ & HGF $>3,020.1 \mathrm{pg} / \mathrm{ml}$ & G-CSF $>477.7 \mathrm{pg} / \mathrm{ml}$ & CRP $>227 \mathrm{mg} / \mathrm{L}$ & Creatinine $>141 \mu \mathrm{mol} / \mathrm{L}$ & Calcium $<1.91 \mathrm{mmol} / \mathrm{L}$ \\
\hline 1 & - & - & - & - & - & - \\
\hline 2 & - & + & + & - & - & + \\
\hline 3 & - & - & - & - & - & - \\
\hline 4 & + & - & - & - & - & b \\
\hline 5 & - & + & + & - & - & $\mathrm{b}$ \\
\hline 6 & - & + & - & + & - & b \\
\hline 7 & - & - & - & - & + & b \\
\hline 8 & - & - & - & - & - & + \\
\hline 9 & - & - & - & - & - & - \\
\hline 10 & + & - & + & - & - & + \\
\hline 11 & - & - & - & - & - & - \\
\hline 12 & + & - & + & - & - & - \\
\hline 13 & - & + & - & - & - & - \\
\hline 14 & + & - & + & - & - & + \\
\hline
\end{tabular}

${ }^{a}$ AP, Acute pancreatitis; CRP, C-reactive protein; G-CSF, Granulocyte colong-stimulating factor; HGF, Hepatocyte growth factor; IL, Interleukin. +, Value above cutoff point (except for calcium below cutoff point). -, Value below cutoff point (except for calcium above cutoff point). ${ }^{\mathrm{b}}$ Missing data. 
Table 7 Diagnostic performances of biomarkers markers in predicting severe acute pancreatitis in patients with admission modified Marshall score $<2(N=142)^{a}$

\begin{tabular}{|c|c|c|c|c|c|c|}
\hline $\begin{array}{l}\text { Predictive marker and } \\
\text { optimal cutoff point }\end{array}$ & AUC & vity (\%) & Specificity (\%) & LR+ & LR- & DOR \\
\hline $\mathrm{IL}-8(>88.1 \mathrm{pg} / \mathrm{ml})$ & 0.79 (0.68 to 0.90$)$ & 25.6 (11.7 to 54.7) & 95.3 (90.2 to 97.8 ) & 6.1 (2.0 to 19.0$)$ & 0.75 (0.54 to 1.05$)$ & 8.1 (2.0 to 33.6) \\
\hline $\mathrm{GF}(>3,020.1 \mathrm{pg} / \mathrm{ml})$ & 0.83 (0.73 to 0.92$)$ & 7 to 54.7$)$ & 90 to 97.8$)$ & 6.1 (2.0 to 19.0$)$ & 54 to 1.05$)$ & B3.6) \\
\hline -CSF (>477.7 pg/ml) & 0.78 (0.65 to 0.90$)$ & 35.6 (16.3 to 61.2) & 96.1 (91.2 to 98.3) & 9.1 (3.0 to 27.7 ) & 0.67 (0.45 to 0.99) & $13.7(3$ \\
\hline CRP $(>227$ mg/L) & 0.54 (0.37 to 0.70$)$ & 7.1 (1.3 to 31.5$)$ & 95.3 (90.2 to 97.8) & 1.5 (0.2 to 11.8 ) & $0.97(0.2$ to 11.8$)$ & $1.6(0.2$ to 14.0$)$ \\
\hline reatining $(>111$ & 0.62 (0.45 to 0.79$)$ & 7.1 (1.3 to 31.5$)$ & 99.2 (95.7 to 99.9) & 9.1 (0.6 to 37.2 ) & 0.94 (0.91 to 1.08) & $9.7(0.57$ to 164.2 \\
\hline Calcium (<1.91 mmol/L) & 0.74 (0.60 to 0.88$)$ & 36.4 (15.2 to 64.6) & 94.3 (88.7 to 97.2 ) & $6.4(2.2$ to 18.5$)$ & 0.68 (0.43 to 1.06$)$ & 9.5 (2.2 to 40.2$)$ \\
\hline $\mathrm{H}-\mathrm{CSF}+\mathrm{IL}-8+\mathrm{HGF}$ & & 57.1 (32.6 to 78.6$)$ & 90.6 (84.3 to 94.6$)$ & $6.1(3.0$ to 12.3$)$ & 0.47 (0.26 to 0.87$)$ & 12.9 (3.8 to 43.4$)$ \\
\hline
\end{tabular}

aL, Interleukin; CRP, C-reactive protein; IL, interleukin; G-CSF, Granulocyte colony-stimulating factor; HGF, Hepatocyte growth factor; IL, Interleukin; AUC, Area under the curve; DOR, Diagnostic odds ratio; LR-, Negative likelihood ratio; LR+, Positive likelihood ratio. 95\% confidence intervals are given in parentheses.

who presented with modified Marshall scores $<2$, HGF, as well as also IL-8 and G-CSF, measured upon admission predicted later development of OD. The combination of HGF, G-CSF and IL- 8 identified 8 of 14 of these severe AP patients. Thus, in 6 of 14 patients, none of these cytokines were raised above the cutoff level, although there was no difference in the clinical course of the disease. The reason for this may derive from variations in immune responses between individuals, the kinetics of the cytokines and/or the phase of AP. This raises the question whether combining cytokines with other, non-cytokine-related prognostic markers might improve the predictive power for severe
AP. Several markers, other than cytokines, have been found to be of prognostic value in OD associated with systemic inflammation [26-30]. Furthermore, novel biomarkers will most probably be discovered by means of systems biology, as reviewed by Skibsted et al. [31].

The crucial question in clinical practice is how to distinguish, upon admission to the hospital, those patients with transient OD from those with persistent OD. At present, no means of identifying such patients exist. In our present study, the limited number of persistent OD patients had higher levels of HGF, IL-6 and IL-8, but the differences were not statistically significant. Whether

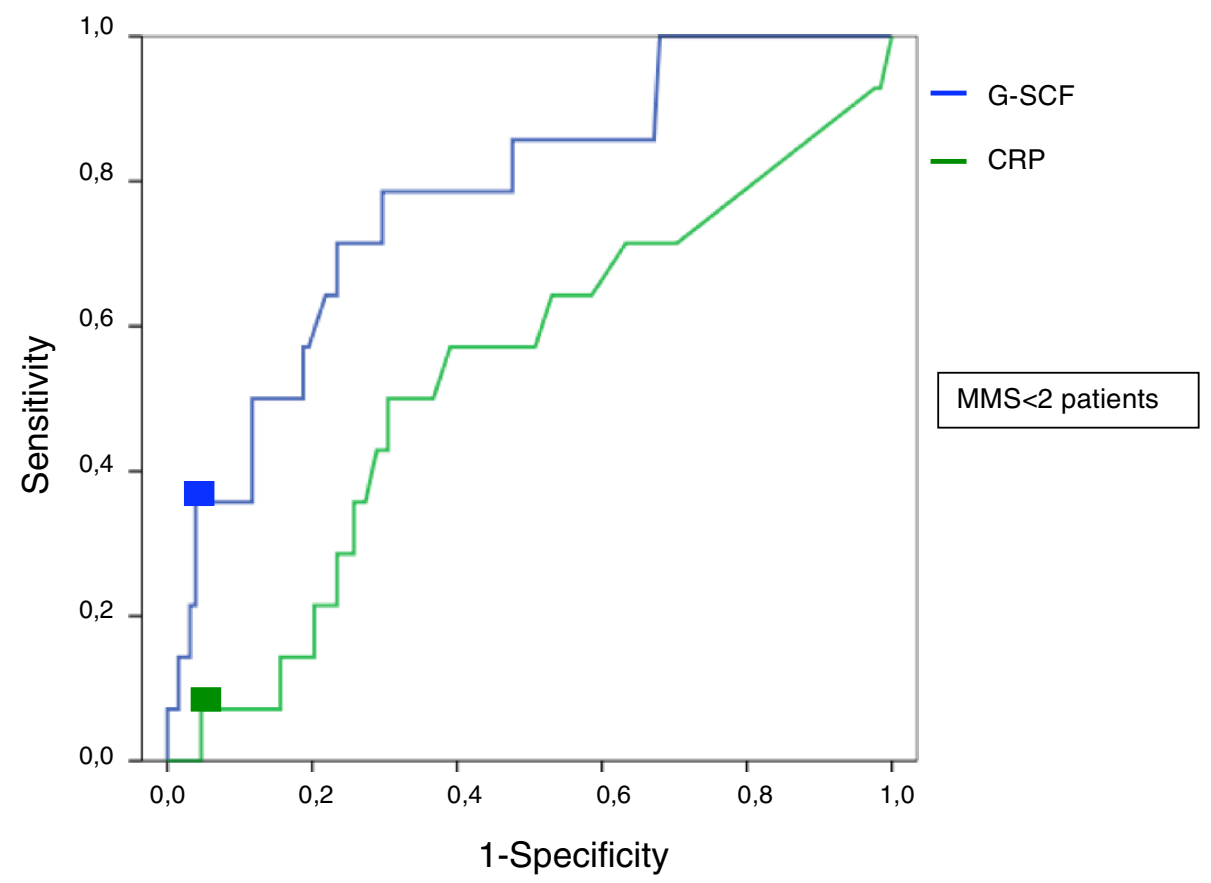

Figure 3 Receiver operating characteristic curves of granulocyte colony-stimulating factor and C-reactive protein for prediction of severe acute pancreatitis in patients with admission modified Marshall scores $<2(\boldsymbol{N}=\mathbf{1 4 2})$. Boxes indicate clinically optimal cutoff points used to calculate the sensitivity and specificity of each biomarker given in Table 7. CRP, C-reactive protein; G-CSF, Granulocyte colony-stimulating factor; MMS, Modified Marshall score. 
these cytokines, as individual or combined markers, can aid in distinguishing between transient and persistent OD needs to be studied in a larger number of OD patients upon admission to the hospital.

Although no specific treatment for AP exists so far, concomitantly with better understanding of the underlying pathophysiology, immunomodulatory treatment has become a matter of interest. However, many patients already have OD upon admission [23]. In addition, AP patients may develop immune suppression rapidly [32]. In such patients, anti-inflammatory therapy may be detrimental. In some patients, immune suppression is confined mainly to the circulation and the inflammatory stage proceeds in other body compartments, such as the lungs [33]. In this complex setting, the determination of immune inflammatory status may aid in the selection of appropriate immunemodulating therapy to prevent or alleviate OD [34].

\section{Conclusions}

We analyzed the levels of 48 cytokines in 163 patients with AP upon admission to the hospital. IL-6 and HGF were independent predictive markers of severe AP; however, neither cytokine nor the combination of them was perfect in identifying AP patients at risk for severe disease. In fact, the results are similar to those for CRP, creatinine and calcium. IL-8, HGF and G-CSF levels could be used to predict severe AP in patients without clinical signs of OD upon admission. To the best of our knowledge, this study is the largest to date with regard to the number of cytokines screened in a group of AP patients. The possibility that combining cytokines with prognostic markers other than cytokines improves the prediction of severe AP warrants further studies.

\section{Key messages}

- The levels of 14/47 cytokines upon admission to the hospital were higher in patients with severe AP than in those with mild or moderately severe AP.

- IL-6 and HGF were independent predictors of severe AP.

- The predictive value of IL-6 6 and HGF was comparable to that of CRP, creatinine and calcium.

- Many patients who will develop severe AP, did not have signs of OD upon admission to hospital.

- IL-8, HGF and G-CSF is able to predict severe AP in patients without clinical signs of OD at the time of hospital admission.

\section{Additional file}

Additional file 1: Diagnostic performances of the 14 cytokines that could differentiate mild or moderately severe AP from severe AP.

\section{Abbreviations}

AP: Acute pancreatitis; AUC: Area under the curve; $\beta$-NGF: $\beta$ nerve growth factor; CRP: C-reactive protein; CTACK: Cutaneous T-cell attracting chemokine; DOR: Diagnostic odds ratio; FGF: Basic fibroblast growth factor; G-CSF: Granulocyte colony-stimulating factor; GM-CSF: Granulocyte-macrophage colony-stimulating factor; HGF: Hepatocyte growth factor; IFN: Interferon; IL: Interleukin; IL-1Ra: Interleukin 1 receptor antagonist; IL-2Ra: Interleukin 2 receptor a; IP-10: Interferon-y-induced protein 10; LIF: Leukemia inhibitory factor; LR: Likelihood ratio; MCP: Monocyte chemotactic protein; M-CSF: Macrophage colony-stimulating factor; MIF: Macrophage migration inhibitory factor; MIG: Monokine induced by interferon $\gamma$; MIP: Macrophage inflammatory protein; OD: Organ dysfunction; PDGF-BB: Platelet-derived growth factor, two BB chains; RANTES: Regulated on activation, normal T cell expressed and secreted; ROC: Receiver operating characteristic curve; SCF: Stem cell factor; SCGF- $\beta$ : Stem cell growth factor $\beta$; SDF: Stromal cell-derived factor; TNF: Tumor necrosis factor; TRAIL: Tumor necrosis factor-related apoptosis-inducing ligand; VEGF: Vascular endothelial growth factor.

\section{Competing interests}

The authors declare that they have no competing interests.

\section{Authors' contributions}

AN and LKyh collected clinical data and participated in data analyses. AN and HR drafted the manuscript. MM analyzed the cytokine levels. PM was a supervisor of the data analyses and participated in designing the study. LKyl, EK and PP participated in designing and coordinating the study and provided supervision. MS and HR designed and supervised the study. All authors critically revised the manuscript and read and approved the final version.

\section{Acknowledgements}

Mrs. Teija Kanasuo is thanked for her excellent technical assistance. The work was supported by the Martti I Turunen Foundation and the Emil Aaltonen Foundation (to AN) and by the Academy of Finland (to MS).

\section{Author details}

'Department of Surgery, Helsinki University Central Hospital, POB 340, Helsinki 00029 HUS, Finland. ${ }^{2}$ MediCity Research Laboratory, University of Turku, Tykistönkatu 6A, 20520 Turku, Finland. ${ }^{3}$ Department of Medical Microbiology and Immunology, University of Turku, Kiinamyllynkatu 10, 20520 Turku, Finland. ${ }^{4}$ Department of Bacteriology and Immunology, Haartman Institute, University of Helsinki, PO Box 21, Helsinki 00014, Finland. ${ }^{5}$ National Institute for Health and Welfare, Turku, Finland.

Received: 18 February 2014 Accepted: 1 May 2014 Published: 21 May 2014

\section{References}

1. Banks PA, Bollen TL, Dervenis C, Gooszen HG, Johnson CD, Sarr MG, Tsiotos GG, Vege SS, Acute Pancreatitis Classification Working Group: Classification of acute pancreatitis-2012: revision of the Atlanta classification and definitions by international consensus. Gut 2013, 62:102-111.

2. Halonen KI, Leppaniemi AK, Puolakkainen PA, Lundin JE, Kemppainen EA, Hietaranta AJ, Haapiainen RK: Severe acute pancreatitis: prognostic factors in 270 consecutive patients. Pancreas 2000, 21:266-271.

3. Gloor B, Müller CA, Worni M, Martignoni ME, Uhl W, Büchler MW: Late mortality in patients with severe acute pancreatitis. Br I Surg 2001, 88:975-979.

4. McKay CJ, Buter A: Natural history of organ failure in acute pancreatitis. Pancreatology 2003, 3:111-114.

5. Vege SS, Gardner TB, Chari ST, Munukuti P, Pearson RK, Clain JE, Petersen BT, Baron TH, Farnell MB, Sarr MG: Low mortality and high morbidity in severe acute pancreatitis without organ failure: a case for revising the Atlanta classification to include "moderately severe acute pancreatitis.". Am J Gastroenterol 2009, 104:710-715.

6. Norman J: The role of cytokines in the pathogenesis of acute pancreatitis. Am J Surg 1998, 175:76-83.

7. Kylänpää ML, Repo H, Puolakkainen PA: Inflammation and immunosuppression in severe acute pancreatitis. World I Gastroenterol 2010, 16:2867-2872. 
8. Buter A, Imrie CW, Carter CR, Evans S, McKay CJ: Dynamic nature of early organ dysfunction determines outcome in acute pancreatitis. Br J Surg 2002, 89:298-302.

9. Johnson CD, Abu-Hilal M: Persistent organ failure during the first week as a marker of fatal outcome in acute pancreatitis. Gut 2004, 53:1340-1344.

10. Banks PA, Freeman ML: Practice guidelines in acute pancreatitis. Am J Gastroenterol 2006, 101:2379-2400.

11. Forsmark CE, Baillie J: AGA Institute technical review on acute pancreatitis. Gastroenterology 2007, 132:2022-2044.

12. Leser HG, Gross V, Scheibenbogen C, Heinisch A, Salm R, Lausen M, Rückauer K, Andreesen R, Farthmann EH, Schölmerich J: Elevation of serum interleukin- 6 concentration precedes acute-phase response and reflects severity in acute pancreatitis. Gastroenterology 1991, 101:782-785.

13. Brivet FG, Emilie D, Galanaud P: Pro- and anti-inflammatory cytokines during acute severe pancreatitis: an early and sustained response, although unpredictable of death. Crit Care Med 1999, 27:749-755.

14. Chen CC, Wang SS, Lee FY, Chang FY, Lee SD: Proinflammatory cytokines in early assessment of the prognosis of acute pancreatitis. Am J Gastroenterol 1999, 94:213-218.

15. Mayer J, Rau B, Gansauge F, Beger HG: Inflammatory mediators in human acute pancreatitis: clinical and pathophysiological implications. Gut 2000, 47:546-552.

16. Marshall JC, Cook DJ, Christou NV, Bernard GR, Sprung CL, Sibbald WJ: Multiple organ dysfunction score: a reliable descriptor of a complex clinical outcome. Crit Care Med 1995, 23:1638-1652.

17. Newcombe RG: Two-sided confidence intervals for the single proportion: comparison of seven methods. Stat Med 1998, 17:857-872.

18. Glas AS, Lijmer JG, Prins MH, Bonsel GJ, Bossuyt PM: The diagnostic odds ratio: a single indicator of test performance. J Clin Epidemiol 2003, 56:1129-1135.

19. Nakamura T, Nishizawa T, Hagiya M, Seki T, Shimonishi M, Sugimura A Tashiro K, Shimizu S: Molecular cloning and expression of human hepatocyte growth factor. Nature 1989, 342:440-443.

20. Aoun E, Chen J, Reighard D, Gleeson FC, Whitcomb DC, Papachristou Gl: Diagnostic accuracy of interleukin- 6 and interleukin- 8 in predicting severe acute pancreatitis: a meta-analysis. Pancreatology 2009, 9:777-785.

21. Ueda T, Takeyama Y, Toyokawa A, Kishida S, Yamamoto M, Saitoh Y: Significant elevation of serum human hepatocyte growth factor levels in patients with acute pancreatitis. Pancreas 1996, 12:76-83.

22. Espinosa L, Linares PM, Bejerano A, Lopez C, Sanchez A, Moreno-Otero R, Gisbert JP: Soluble angiogenic factors in patients with acute pancreatitis. J Clin Gastroenterol 2011, 45:630-637.

23. Johnson CD, Kingsnorth AN, Imrie CW, McMahon MJ, Neoptolemos JP, McKay C, Toh SK, Skaife P, Leeder PC, Wilson P, Larvin M, Curtis LD: Double blind, randomised, placebo controlled study of a platelet activating factor antagonist, lexipafant, in the treatment and prevention of organ failure in predicted severe acute pancreatitis. Gut 2001, 48:62-69.

24. Harrison DA, D'Amico G, Singer M: Case mix, outcome, and activity for admissions to UK critical care units with severe acute pancreatitis: a secondary analysis of the ICNARC Case Mix Programme Database. Crit Care 2007, 11:S1

25. Wu BU, Conwell DL: Update in acute pancreatitis. Curr Gastroenterol Rep 2010, 12:83-90.

26. Oiva J, Mustonen $H$, Kylänpää ML, Kyhälä L, Kuuliala K, Siitonen S, Kemppainen E, Puolakkainen $\mathrm{P}$, Repo $\mathrm{H}$ : Acute pancreatitis with organ dysfunction associates with abnormal blood lymphocyte signaling: controlled laboratory study. Crit Care 2010, 14:R207.

27. Chakraborty S, Kaur S, Muddana V, Sharma N, Wittel UA, Papachristou Gl, Whitcomb D, Brand RE, Batra SK: Elevated serum neutrophil gelatinaseassociated lipocalin is an early predictor of severity and outcome in acute pancreatitis. Am J Gastroenterol 2010, 105:2050-2059.

28. Yilmaz G, Köksal I, Karahan SC, Mentese A: The diagnostic and prognostic significance of soluble urokinase plasminogen activator receptor in systemic inflammatory response syndrome. Clin Biochem 2011, 44:1227-1230.

29. Moore DJ, Greystoke A, Butt F, Wurthner J, Growcott J, Hughes A, Dive C: A pilot study assessing the prognostic value of CK18 and nDNA biomarkers in severe sepsis patients. Clin Drug Investig 2012, 32:179-187.

30. Oiva J, Mustonen H, Kylänpää ML, Kuuliala K, Siitonen S, Kemppainen E, Puolakkainen $\mathrm{P}$, Repo H: Patients with acute pancreatitis complicated by organ dysfunction show abnormal peripheral blood polymorphonuclear leukocyte signaling. Pancreatology 2013, 13:118-124.

31. Skibsted S, Bhasin MK, Aird WC, Shapiro Nl: Bench-to-bedside review: future novel diagnostics for sepsis-a systems biology approach. Crit Care 2013, 17:231.

32. Kylänpää-Bäck ML, Takala A, Kemppainen E, Puolakkainen $\mathrm{P}$, Kautiainen $\mathrm{H}$, Jansson SE, Haapiainen R, Repo H: Cellular markers of systemic inflammation and immune suppression in patients with organ failure due to severe acute pancreatitis. Scand J Gastroenterol 2001, 36:1100-1107.

33. Cavaillon JM, Annane D: Compartmentalization of the inflammatory response in sepsis and SIRS. J Endotoxin Res 2006, 12:151-170.

34. Caldwell CC, Hotchkiss RS: The first step in utilizing immune-modulating therapies: immune status determination. Crit Care 2011, 15:108.

doi:10.1186/cc13885

Cite this article as: Nieminen et al: Circulating cytokines in predicting development of severe acute pancreatitis. Critical Care 2014 18:R104.

\section{Submit your next manuscript to BioMed Central and take full advantage of:}

- Convenient online submission

- Thorough peer review

- No space constraints or color figure charges

- Immediate publication on acceptance

- Inclusion in PubMed, CAS, Scopus and Google Scholar

- Research which is freely available for redistribution
C) BioMed Central 